\title{
T1p Magnetic Resonance Imaging to Assess Cartilage Damage After Primary Shoulder Dislocation
}

\author{
Vishal Saxena, MD ${ }^{1}$, Kevin D'Aquilla, BS ${ }^{2}$, Shannon Marcoon, BA ${ }^{1}$, Guruprasad \\ Krishnamoorthy, BE, MSc ${ }^{2}$, Joshua A. Gordon, MD ${ }^{1}$, James L. Carey, MD, MPH ${ }^{1}$, Ari \\ Borthakur, PhD, MBA ${ }^{2}$, J. Bruce Kneeland, MD², John D. Kelly IV, MD ${ }^{1}$, Ravinder Reddy, \\ $\mathrm{PhD}^{2}$, and Brian J. Sennett, $\mathbf{M D}^{1}$ \\ ${ }^{1}$ Penn Sports Medicine Center, University of Pennsylvania, Philadelphia, PA, USA \\ ${ }^{2}$ Center for Magnetic Resonance and Optical Imaging, Department of Radiology, University of \\ Pennsylvania, Philadelphia, PA, USA
}

\begin{abstract}
Background-Patients who suffer anterior shoulder dislocations are at higher risk of developing glenohumeral arthropathy, but little is known about the initial cartilage damage after a primary shoulder dislocation. T1 $\rho$ is a magnetic resonance imaging (MRI) technique that allows quantification of cartilage proteoglycan content and can detect physiologic changes in articular cartilage.
\end{abstract}

\begin{abstract}
Purpose-To establish baseline T1 $\rho$ MRI values for glenoid and humeral head cartilage, determine if T1 $\rho$ MRI can detect glenohumeral cartilage damage following traumatic primary shoulder dislocation, and assess for patterns in cartilage damage in anterior shoulder dislocation.
\end{abstract}

Study Design-Prospective cohort study

Methods-Nine male patients (mean age 32.0 years, range 20-59) who sustained first-time anterior shoulder dislocations underwent 3T T1 $\rho$ MRI. Five healthy controls (mean age 27.4 years, range 24-30) without prior dislocation or glenohumeral arthritis also underwent 3T T1 $\rho$ MRI. The T1 $\rho$ relaxation constant was determined for the entire glenoid and humeral head for dislocation patients and healthy controls. The glenoid and humeral head were divided into nine zones, and T1 $\rho$ values were determined for each zone in dislocated and control shoulders to identify patterns in cartilage damage in dislocated shoulders.

Results-Average overall T1 $\rho$ values for humeral head cartilage in dislocated shoulders were significantly greater than controls $(41.7 \mathrm{~ms} \pm 3.9$ versus $38.4 \mathrm{~ms} \pm 0.6$, respectively; $\mathrm{p}=0.03)$. However, average overall T1 $\rho$ values for glenoid cartilage were not significantly different in dislocated shoulders compared to controls $(44.0 \mathrm{~ms} \pm 3.3$ versus $44.6 \mathrm{~ms} \pm 2.4$, respectively; $\mathrm{p}=$ 0.40 ), suggesting worse damage to humeral head cartilage. T1 $\rho$ values in the posterior-middle humeral head were higher in dislocation patients compared to controls $(41.5 \mathrm{~ms} \pm 3.8$ versus $38.2 \mathrm{~ms} \pm 2.2$, respectively; $\mathrm{p}=0.021$ ) and trended toward significance in the posterior-superior

Corresponding author: Brian J. Sennett, MD, Penn Sports Medicine Center, Weightman Hall, 235 S. $33^{\text {rd }}$ St., Philadelphia, PA 19104, brian.sennett@uphs.upenn.edu.

We declare that we have no conflicts of interest in the authorship or publication of this contribution. 
and middle-superior zones $(35.2 \mathrm{~ms} \pm 4.9$ versus $31.3 \mathrm{~ms} \pm 1.0$ and $33.7 \mathrm{~ms} \pm 5.0$ versus $30.5 \mathrm{~ms}$ \pm 1.3 , respectively; $\mathrm{p}=0.056$ ). These three humeral head zones are where Hill-Sachs lesions predominate. T1 $\rho$ values in the anterior-inferior glenoid zone trended toward significance in dislocated patients compared to controls $(47.4 \mathrm{~ms} \pm 5.0$ versus $43.5 \mathrm{~ms} \pm 3.5$, respectively; $\mathrm{p}=$ $0.073)$.

Conclusion-Humeral head cartilage sustained greater damage than glenoid cartilage in primary dislocation. T1 $\rho$ values were higher in glenohumeral zones associated with Bankart and Hill-Sachs lesions. Widespread initial cartilage damage may predispose patients to glenohumeral arthropathy.

\section{Keywords}

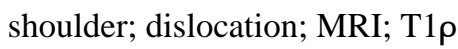

\section{Introduction}

Anterior shoulder dislocation is a common injury, with an estimated incidence of 23.1 dislocations per 100,000 person-years requiring closed reduction (manipulation with or without conscious sedation or general anesthesia) ${ }^{8}$. In anterior dislocations, associated injuries typically involve the anteroinferior glenoid (Bankart and its variant lesions) ${ }^{13}$ and the posterosuperior humeral head (Hill-Sachs lesions) ${ }^{5}$. Patients who suffer a shoulder dislocation are at higher risk of developing glenohumeral arthropathy; this risk increases with age at the time of primary dislocation and recurrent dislocations ${ }^{6}$. However, little is known about the initial cartilage damage after a primary shoulder dislocation.

Magnetic resonance (MR) is an imaging modality that allows improved visualization of intra-articular structures and soft tissues. Advances in field strength and coil design have improved the sensitivity of MR in detecting morphologic changes in cartilage structure. Hayes et al used conventional MRI to detect glenohumeral articular cartilage lesions and found overall sensitivity and specificity of $87.2 \%$ and $80.6 \%$, respectively, compared to arthroscopy ${ }^{4}$. Newer imaging sequences that focus on proteoglycan content and collagen orientation and concentration enable detection of the early changes in cartilage microstructure that precede gross morphologic changes seen with conventional MR sequences. These techniques include T2 mapping, delayed gadolinium-enhanced MR imaging of cartilage (dGEMRIC), and T1p.

T2 mapping relies on the orientation of collagen fibers. Highly organized orientation in the superficial and radial zones results in shorter T2 values, whereas random collagen orientation in the intermediate zone produces longer $\mathrm{T} 2$ values. Thus, changes in $\mathrm{T} 2$ values can signal changes in collagen structure and content. Maizlin et al performed T2 mapping on 27 shoulders and found that areas with higher T2 values correlated with areas of cartilage defects seen on routine MRI ${ }^{9}$. dGEMRIC relies on the negative charge of proteoglycan molecules. Proteoglycans are depleted in damaged cartilage. The negatively charged gadolinium replaces proteoglycans. Measuring T1 relaxation time after administration of intravenous gadolinium enables mapping of areas of damaged cartilage and proteoglycan loss. dGEMRIC has been used to map glenohumeral cartilage in normal shoulders ${ }^{1}$. 
However, a disadvantage of this technique is that it requires a double dose of intravenous gadolinium.

T1 $\rho$ MRI relies on low frequency interactions between hydrogen found in water molecules and proteoglycans. In T1 $\rho$ MRI, a long duration, low power radiofrequency (RF) pulse, also known as the "spin-lock" pulse, is applied to the magnetization in the transverse plane. This spin-locked magnetization relaxes with a time constant (T1 $\rho)$. Higher T1 $\rho$ values correlate with decreased proteoglycan content and, hence, cartilage damage ${ }^{2}$. T1 $\rho$ MRI does not require intravenous contrast and has been shown to detect differences in knee cartilage between Kellgren-Lawrence grades ${ }^{18}$ as well as acetabular cartilage changes in patients with femoroacetabular impingement ${ }^{11}$. Compared to T2 mapping, T1 $\rho$ MRI has been shown to be more sensitive in detecting cartilage degeneration ${ }^{12}$ and offers higher dynamic range and increased tolerance to magic angle effects, which can cause artifacts when imaging cartilage $^{10}$.

The purposes of this prospective pilot study were to: 1) establish baseline T1 $\rho$ MRI values for glenoid and humeral head cartilage; 2) determine if T1 $\rho$ MRI can detect changes in proteoglycan content of glenohumeral cartilage indicating damage following traumatic primary shoulder dislocation; and 3) assess for patterns and preferential locations of glenoid and humeral head cartilage damage in anterior shoulder dislocation.

\section{Materials and Methods}

This study was approved and conducted following the guidelines set forth by our Institutional Review Board. Nine male patients (mean age 32.0 years, range 20-59) who sustained primary anterior shoulder dislocations underwent 3T T1 $\rho$ MRI. Eight patients had their MRI within two weeks of injury. The other patient underwent MRI seven weeks after injury. Seven patients were Caucasian, one patient was African-American, and one patient was Hispanic. Patients were included in this study if they had had no prior shoulder dislocations and were willing and able to undergo MRI evaluation. Exclusion criteria included prior history of glenohumeral arthritis or dysplasia or prior surgery about the shoulder. Five male healthy controls (mean age 27.4 years, range 24-30) without prior shoulder dislocation or glenohumeral arthritis also underwent T1 $\rho$ MRI for comparison.

All scans were conducted on a Siemens Tim Trio (Siemens, Erlangen, Germany) 3T MRI scanner using a product shoulder receive-only coil. Conventional MRI and T1 $\rho$ imaging were performed on all patients. Shimming was conducted on the area of interest, including the glenohumeral compartment. Eight oblique coronal slices of T1 $\rho$ imagery were acquired perpendicular to the glenoid fossa using a magnetically-prepared 3D GRE readout and fat suppression. Imagery was acquired at spin-lock times of 0, 10, 20, 30, and 40 milliseconds $(\mathrm{ms})$. These spin-lock times were chosen as a compromise between total scan time and data fitting accuracy, as we expected our T1 $\rho$ map values to lie within the range of $30-50 \mathrm{~ms}$ based on prior data for cartilage ${ }^{2}$. T1 $\rho$ images were acquired with two averages. Parameters were as follow: spin-lock amplitude $\left(\mathrm{B}_{1}\right.$ power $)=500 \mathrm{~Hz}$, inner loop time to repetition (TR) $=9.1 \mathrm{~ms}$, time to echo $(\mathrm{TE})=4.5 \mathrm{~ms}$, shot TR $=6 \mathrm{~s}$, bandwidth $=400 \mathrm{~Hz}$, flip angle $=10^{\circ}$, 
field of view $(\mathrm{FOV})=140 \times 140 \mathrm{~mm}$, matrix size $=256 \times 256(0.54 \times 0.54 \mathrm{~mm}$ resolution $)$, and slice thickness $=3 \mathrm{~mm}$. Total acquisition time was approximately 12 minutes.

Images were processed offline utilizing purpose-written MATLAB (MathWorks, Inc., Natick, MA) scripts. For each slice, data acquired at each spin-lock time was fitted voxel-byvoxel utilizing a mono-exponential fitting algorithm corrected for steady state phenomena at long spin-lock times, based on the formula

$$
S=S_{0} e^{\left(-t_{s l} / T_{1 \rho}\right)}+C
$$

where $\mathrm{S}$ is the observed signal, $\mathrm{S}_{0}$ is the initial signal with no spin-lock pulse, $\mathrm{t}_{\mathrm{sl}}$ is the applied spin-lock pulse duration, $\mathrm{T}_{1 \rho}$ is the characteristic $\mathrm{T} 1 \rho$ value, and $\mathrm{C}$ is the steady state correction factor.

This procedure generated oblique coronal maps of T1 $\rho$ values. These maps were then manually segmented by an operator based on masks drawn on the raw T1 $\rho$ images, and a plane was defined slice-by-slice by connecting the ends of the segmented cartilage with a drawn line. Using a MATLAB script, the resulting planes were then averaged, and the segmented maps were rotated in $3 \mathrm{D}$ space $90^{\circ}$ around this average plane. The resulting $3 \mathrm{D}$ dataset was then orthographically projected normal to the average plane, averaging T1 $\rho$ values through the whole cartilage depth. This procedure generated a single oblique sagittal map of average T1 $\rho$ values for both the glenoid fossa and humeral head for each subject studied.

T1 $\rho$ values were determined for the entire glenoid and humeral head for each dislocation patient and healthy control. In addition, the glenoid and humeral head were manually divided into nine zones of equal size. The anterior-posterior and superior-inferior widths of the glenoid and humeral head cartilage were divided into thirds, and nine zones were fitted to include the maximal area of cartilage while excluding areas without cartilage (T1 $\rho$ values of $0 \mathrm{~ms})$. Zones were named first in the anterior-posterior direction and then in the superiorinferior direction (Fig. 1). Average T1 $\rho$ values for each zone in both the healthy controls and the patients who had sustained dislocation were compared to assess for differences between the two groups and to identify any patterns in cartilage damage in anterior shoulder dislocation.

All values are reported as mean ( \pm standard deviation (SD)). All statistical analysis was performed using GraphPad Prism (La Jolla, CA). Overall T1 $\rho$ values (the average of all values across the entire glenoid or humeral head face) and regional values for the nine zones for glenoid and humeral head cartilage between healthy controls and dislocation patients were compared using a one-tailed Mann-Whitney U test. The Kruskal-Wallis test was used to make comparisons of $\mathrm{T} 1 \rho$ values from region to region in glenoid or humeral head cartilage. 


\section{Results}

The average overall T1 $\rho$ value for glenoid cartilage across the entire surface was not significantly different in dislocated shoulders compared to control shoulders $(44.0 \mathrm{~ms} \pm 3.3$ versus $44.6 \mathrm{~ms} \pm 2.4$, respectively; $\mathrm{p}=0.40$ ). However, the average overall $\mathrm{T} 1 \rho$ value for humeral head cartilage in dislocated shoulders was significantly greater than controls ( $41.7 \mathrm{~ms} \pm 3.9$ versus $38.4 \mathrm{~ms} \pm 0.6$, respectively; $\mathrm{p}=0.03$ ), suggesting worse damage to humeral head cartilage than glenoid cartilage.

Regional T1 $\rho$ values in healthy controls ranged from $37.6 \mathrm{~ms}$ to $58.3 \mathrm{~ms}$ for glenoid cartilage and $29.2 \mathrm{~ms}$ to $50.5 \mathrm{~ms}$ for humeral head cartilage. Regional $\mathrm{T} 1 \rho$ values in dislocation patients ranged from $35.7 \mathrm{~ms}$ to $60.0 \mathrm{~ms}$ for glenoid cartilage and $28.1 \mathrm{~ms}$ to $60.7 \mathrm{~ms}$ for humeral head cartilage. Figure 2 shows the average T1 $\rho$ value for each of the nine zones in glenoid cartilage in healthy controls (Fig. 2A) and dislocation patients (Fig. 2B). Figure 3 shows the average $\mathrm{T} 1 \rho$ value for each of the nine zones in humeral head cartilage in healthy controls (Fig. 3A) and dislocation patients (Fig. 3B). T1 $\rho$ values in inferior humeral head zones in control shoulders were higher than those in superior zones $(p<0.05)$. Although no glenoid zone in dislocated shoulders had significantly higher T1 $\rho$ values compared to controls ( $p>0.05$ ), dislocated shoulders had higher T1 $\rho$ values in the anterior-inferior glenoid zone which trended toward significance $(\mathrm{p}=0.073)$. This zone is the typical location for capsulolabral lesions (i.e. Bankart lesions) that are common sequelae of anterior shoulder dislocations ${ }^{13}$.

For humeral head cartilage, the average T1 $\rho$ value in the posterior-middle zone was significantly greater in dislocated patients compared to controls $(\mathrm{p}=0.021)$. The middlesuperior and posterior-superior humeral head zones showed a trend of higher T1 $\rho$ values without significance for dislocated patients compared to controls ( $\mathrm{p}=0.056$ for both). Clustered together, these zones correspond to the usual location for Hill-Sachs lesions ${ }^{5}$. All patients were found to have Hill-Sachs lesions. There was no correlation between humeral head T1 $\rho$ values and Hill-Sachs lesion size, amount of bony damage or edema, or age of the patient. The other humeral head zones had statistically similar T1 $\rho$ values in dislocated shoulders compared to controls.

Figure 4 shows a representative T1 $\rho$ coronal slice from each control shoulder. Figure 5 shows a representative T1 $\rho$ coronal slice from each dislocated shoulder. Some patients were found to have large increases in overall T1 $\rho$ values (greater than two standard deviations above those for healthy controls), suggesting severe glenohumeral cartilage damage after dislocation. One out of nine dislocation patients was found to have severe diffuse glenoid cartilage damage with the worst damage in the anterior-inferior and middle-inferior zones (typical for Bankart lesions) as well as the anterior-superior zone. Six out of nine patients sustained severe humeral head cartilage damage. The rest had T1 $\rho$ values similar to controls, signifying little to no cartilage damage.

Coronal slices were reformatted to generate oblique sagittal T1 $\rho$ maps, which further highlighted the variability in baseline glenohumeral $\mathrm{T} 1 \rho$ values for healthy controls and the cartilage damage in dislocated shoulders. Figure 6 shows representative glenoid and humeral 
head T1 $\rho$ maps for one healthy control shoulder. Figure 7 shows glenoid and humeral head T1 $\rho$ maps for a dislocation patient who sustained severe damage to humeral head cartilage.

\section{Discussion}

T1 $\rho$ is an emerging MRI technique that can be used to detect early signs of degeneration of articular cartilage before these changes become apparent on gross inspection or plain radiographs. The purpose of the present study was threefold: 1) establish baseline T1 $\rho$ MRI values for glenoid and humeral head cartilage; 2) determine if T1 $\rho$ MRI demonstrates cartilage damage following anterior shoulder dislocation; and 3) assess for patterns or preferential glenohumeral cartilage damage in anterior shoulder dislocation.

The average overall $\mathrm{T} 1 \rho$ value in control shoulders ranged from $41.9 \mathrm{~ms}$ to $46.9 \mathrm{~ms}$ for glenoid cartilage and $37.7 \mathrm{~ms}$ to $39.1 \mathrm{~ms}$ for humeral head cartilage. While there was little difference in T1 $\rho$ values across zones in glenoid cartilage, inferior humeral head cartilage tended to have higher T1 $\rho$ values than superior zones in healthy controls. Previously, La Rocca Vieira et al performed T1 $\rho$ MRI mapping in four healthy controls to determine baseline T1 $\rho$ values for glenoid and humeral head cartilage ${ }^{7}$. They found baseline T1 $\rho$ values of $32.4-36.9 \mathrm{~ms}$ for glenoid cartilage and $37.9-48.5 \mathrm{~ms}$ in humeral head cartilage. La Rocca Vieira et al observed lower baseline T1 $\rho$ values for glenoid cartilage than our data likely due to differences in their T1 $\rho$ pulse sequence and use of a spin-lock amplitude of $250 \mathrm{~Hz}$, compared to $500 \mathrm{~Hz}$ in our study. These differences may explain the lower baseline glenoid cartilage T1 $\rho$ values in their study compared to ours. Another major difference in our results compared to those of La Rocca Vieira et al is that our study found higher baseline T1 $\rho$ values in glenoid cartilage compared to humeral head cartilage. This is likely because La Rocca Vieira et al acquired T1 $\rho$ data based on axial MRI sequences, which limit data acquisition from the superior zones of humeral head cartilage due to the marked curvature relative to the axial plane and the finite thickness of the slices. This limit on data acquisition based on slice orientation is caused by the medical imaging phenomenon of partial voluming and spillover in which signal losses or gains in imaging voxels are due to the finite resolution of the scanner and in vivo tissue boundaries. Our method acquires the image data in the oblique coronal plane which allows more accurate imaging in the superior humeral head zones, which were found to have lower T1 $\rho$ values. Thus, our average humeral head T1 $\rho$ values were lower because we analyzed data from almost the entire humeral head cartilage region of interest, excluding only slices in the extreme anterior or inferior aspects.

Recurrent dislocations and instability are a risk factor for the development of glenohumeral arthropathy. In their landmark study, Hovelius et al prospectively followed patients who had sustained first-time anterior shoulder dislocation and found that after 25 years, 39\% of patients with recurrent dislocations developed moderate to severe glenohumeral arthropathy. However, even patients with just a single dislocation without recurrence are at risk, with $18 \%$ developing moderate/severe glenohumeral arthropathy ${ }^{6}$.

Our study findings are consistent with the long-term findings of Hovelius et al. Some patients sustained little to no glenohumeral cartilage damage after a primary shoulder dislocation. In contrast, several patients had markedly elevated $\mathrm{T} 1 \rho$ values indicative of 
severe cartilage damage. There was no significant increase in average overall glenoid T1 $\rho$ values for dislocation patients compared to controls. However, there was a significant increase in average overall $\mathrm{T} 1 \rho$ values for humeral head cartilage in dislocation patients, suggesting that anterior shoulder dislocation tends to preferentially damage humeral cartilage rather than glenoid cartilage. This point is further supported by the finding that $6 / 9$ dislocation patients sustained severe humeral head cartilage damage (T1 $\rho$ values greater than two standard deviations above those for healthy controls), whereas only $1 / 9$ patients sustained severe glenoid cartilage damage.

Glenoid and humeral heads were further subdivided into nine zones to assess for any patterns in cartilage damage. None of the nine glenoid zones had statistically significant increases in T1 $\rho$ values compared to their respective zones in control shoulders. However, T1 $\rho$ values in the anterior-inferior glenoid zone showed a near significant increase in dislocated shoulders compared to controls $(\mathrm{p}=0.073)$. This finding is consistent with the anteroinferior glenoid being the most common site for Bankart lesions after anterior shoulder dislocation ${ }^{13}$ and suggests that dislocation can also damage the cartilage in the anteroinferior glenoid. Overall, we found that the glenoid cartilage was relatively spared from damage. The one patient with severe glenoid damage was found to have diffuse damage, particularly in the anterior-inferior and middle-inferior zones (the typical location for Bankart lesions) as well as in the anterior-superior zone.

Although the reason for the relative lack of glenoid cartilage damage is unknown, one possible explanation is that the labrum may shield the underlying cartilage from major traumatic injury. The lack of consistent, widespread glenoid cartilage damage, even in central zones, may be reassuring and may explain why the majority of patients after single dislocation do not go on to developing severe glenohumeral arthropathy. However, as multiple dislocations predispose patients to glenohumeral arthropathy, perhaps through worsening glenoid cartilage damage, the relative lack of glenoid cartilage damage after the first dislocation may prompt consideration of earlier operative stabilization to protect glenoid cartilage from damage in further dislocations.

Regarding humeral head cartilage, the posterior-middle humeral head zone had significantly higher T1 $\rho$ values in dislocated shoulders compared to controls. There was also a trend toward higher T1 $\rho$ values in the middle-superior and posterior-superior humeral head zones $(p=0.056)$, suggestive of cartilage damage in these zones. Together, these three zones are the usual location of Hill-Sachs lesions ${ }^{5}$. As all patients were found to have Hill-Sachs lesions, our T1 $\rho$ maps confirm the presence of Hill-Sachs lesions in the posterosuperior humeral head and did not identify other consistent, occult humeral head lesions which should be associated with anterior shoulder dislocation. Although recurrence, age greater than 25 at the time of initial dislocation, high energy sports, and alcohol abuse have been shown to increase the risk of post-dislocation glenohumeral arthropathy ${ }^{6}$, to our knowledge, no study has determined whether the presence or size of Hill-Sachs lesions correlates with the development of subsequent arthritis. However, presence of large Hill-Sachs lesions may lead to recurrent instability ${ }^{14,17}$, particularly with the shoulder in abduction and external rotation when the Hill-Sachs lesion engages with the glenoid. In addition to having HillSachs lesions, some patients had more widespread humeral head cartilage damage as well. 
When considering all patients together, there were no other significant trends or patterns in cartilage damage aside from consistent damage in the region associated with Hill-Sachs lesions. However, the patients who sustained severe humeral head damage on top of that associated with their Hill-Sachs lesion may be at higher risk of developing glenohumeral arthritis in the future.

This study has several limitations. Our sample size was relatively small and homogeneous, limiting the generalizability of the study. However, this study was designed to be a pilot study demonstrating the sensitivity and feasibility of T1 $\rho$ MRI in detecting cartilage damage following anterior shoulder dislocation. Although we have shown that T1 $\rho$ can detect cartilage damage following primary shoulder dislocation, larger studies with longer followup are needed to adequately detect any patterns in glenohumeral cartilage damage long-term and across genders and ethnicities. Our cohort of patients will be followed to determine if any initial cartilage damage improves or worsens over time. Our study also did not have arthroscopic correlation of cartilage damage. However, since not all patients who sustain shoulder dislocation undergo operative stabilization, subjecting patients to unnecessary arthroscopy would be unethical.

Most prior studies using T1 $\rho$ MRI have examined knee joint cartilage ${ }^{3,15,16}$. Although others have imaged glenohumeral cartilage using T2 mapping ${ }^{9}$, our study, along with that of La Rocca Vieira et $\mathrm{al}^{7}$, is among the first to image glenohumeral cartilage with T1 $\rho$ MRI. Furthermore, to our knowledge, our study is the first to use biochemical MRI sequences to evaluate glenohumeral cartilage damage after anterior shoulder dislocation. Thus, this study represents a novel application of an emerging MRI technique to answer an important clinical question.

In conclusion, T1 $\rho$ MRI is sensitive for detecting glenohumeral cartilage damage following anterior shoulder dislocation. In general, humeral head cartilage sustained greater damage than glenoid cartilage. Some patients had only minimal to no cartilage damage. However, several patients were found to have severe glenohumeral cartilage damage. Compared to healthy controls, dislocation patients had higher T1 $\rho$ values in the anterior-inferior glenoid and posterior-middle, posterior-superior, and middle-superior humeral head, the typical locations of Bankart and Hill-Sachs lesions, respectively. These lesions are common sequelae of anterior shoulder dislocation. In addition to damage in these expected regions, several patients had more widespread glenohumeral cartilage damage. Indeed, the variability in cartilage damage observed in this study may prove to be the most important risk factor in developing glenohumeral arthropathy.

\section{References}

1. Bittersohl B, Miese FR, Dekkers C, et al. T2* mapping and delayed gadolinium-enhanced magnetic resonance imaging in cartilage (dGEMRIC) of glenohumeral cartilage in asymptomatic volunteers at 3 T. Eur Radiol. 2013; 23(5):1367-1374. [PubMed: 23179527]

2. Borthakur A, Mellon E, Niyogi S, et al. Sodium and T1rho MRI for molecular and diagnostic imaging of articular cartilage. NMR Biomed. 2006; 19(7):781-821. [PubMed: 17075961]

3. Goto H, Iwama Y, Fujii M, et al. A preliminary study of the T1rho values of normal knee cartilage using 3T-MRI. Eur J Radiol. 2012; 81(7):e796-803. [PubMed: 22525597] 
4. Hayes ML, Collins MS, Morgan JA, Wenger DE, Dahm DL. Efficacy of diagnostic magnetic resonance imaging for articular cartilage lesions of the glenohumeral joint in patients with instability. Skeletal Radiol. 2010; 39(12):1199-1204. [PubMed: 20411385]

5. Hill HA, Sachs MD. The Grooved Defect of the Humeral Head. Radiology. 1940; 35(6):690-700.

6. Hovelius L, Saeboe M. Neer Award 2008: Arthropathy after primary anterior shoulder dislocation--223 shoulders prospectively followed up for twenty-five years. J Shoulder Elb Surg. 2009; 18(3):339-347.

7. La Rocca Vieira R, Pakin SK, de Albuquerque Cavalcanti CF, Schweitzer M, Regatte R. Threedimensional spin-lock magnetic resonance imaging of the shoulder joint at $3 \mathrm{~T}$ : initial experience. Skeletal Radiol. 2007; 36(12):1171-1175. [PubMed: 17912522]

8. Leroux T, Wasserstein D, Veillette C, et al. Epidemiology of primary anterior shoulder dislocation requiring closed reduction in Ontario, Canada. Am J Sports Med. 2014; 42(2):442-450. [PubMed: 24275862]

9. Maizlin ZV, Clement JJ, Patola WB, et al. T2 mapping of articular cartilage of glenohumeral joint with routine MRI correlation--initial experience. HSS J. 2009; 5(1):61-66. [PubMed: 19159984]

10. Nozaki T, Kaneko Y, Yu HJ, et al. T1rho mapping of entire femoral cartilage using depth- and angle-dependent analysis. Eur Radiol. 2015

11. Rakhra KS, Lattanzio PJ, Cárdenas-Blanco A, Cameron IG, Beaulé PE. Can T1-rho MRI detect acetabular cartilage degeneration in femoroacetabular impingement?: a pilot study. J Bone Joint Surg Br. 2012; 94(9):1187-1192. [PubMed: 22933489]

12. Regatte RR, Akella SVS, Lonner JH, Kneeland JB, Reddy R. T1rho relaxation mapping in human osteoarthritis (OA) cartilage: comparison of T1rho with T2. J Magn Reson Imaging. 2006; 23(4): 547-553. [PubMed: 16523468]

13. Rowe CR, Patel D, Southmayd WW. The Bankart procedure: a long-term end-result study. J Bone Joint Surg Am. 1978; 60(1):1-16. [PubMed: 624747]

14. Rowe CR, Zarins B, Ciullo JV. Recurrent anterior dislocation of the shoulder after surgical repair. Apparent causes of failure and treatment. J Bone Joint Surg Am. 1984; 66(2):159-168. [PubMed: 6693441]

15. Singh A, Haris M, Cai K, et al. High resolution T1 $\rho$ mapping of in vivo human knee cartilage at 7T. PloS One. 2014; 9(5):e97486. [PubMed: 24830386]

16. Souza RB, Feeley BT, Zarins ZA, et al. T1rho MRI relaxation in knee OA subjects with varying sizes of cartilage lesions. The Knee. 2013; 20(2):113-119. [PubMed: 23159719]

17. Voos JE, Livermore RW, Feeley BT, et al. Prospective evaluation of arthroscopic bankart repairs for anterior instability. Am J Sports Med. 2010; 38(2):302-307. [PubMed: 20028847]

18. Wang L, Chang G, Xu J, et al. T1rho MRI of menisci and cartilage in patients with osteoarthritis at 3T. Eur J Radiol. 2012; 81(9):2329-2336. [PubMed: 21908122] 

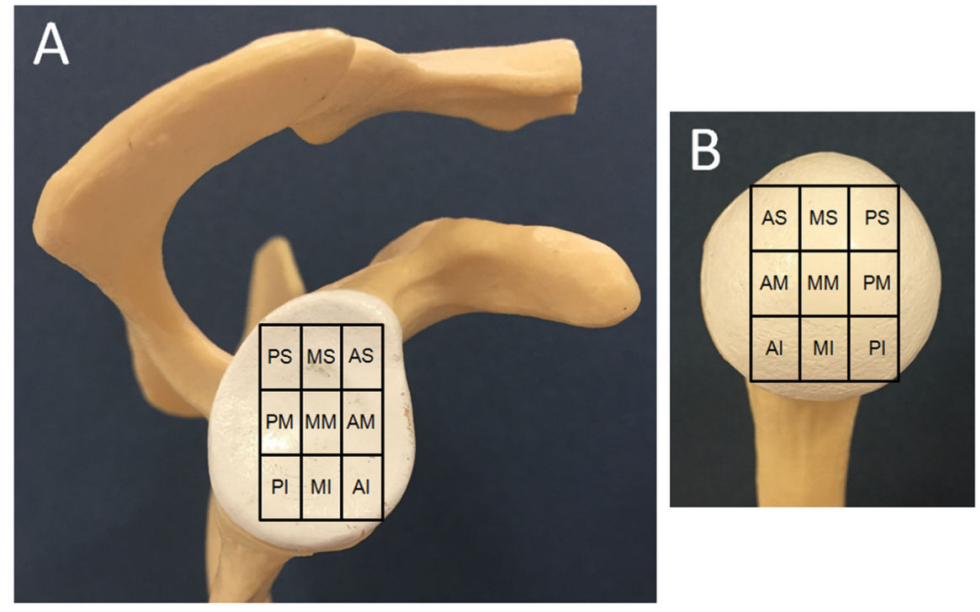

Figure 1.

The nine zones for glenoid (A) and humeral head (B) cartilage. Anterior-superior (AS), anterior-middle (AM), anterior-inferior (AI), middle-superior (MS), middle-middle (MM), middle-inferior (MI), posterior-superior (PS), posterior-middle (PM), and posterior-inferior (PI). 

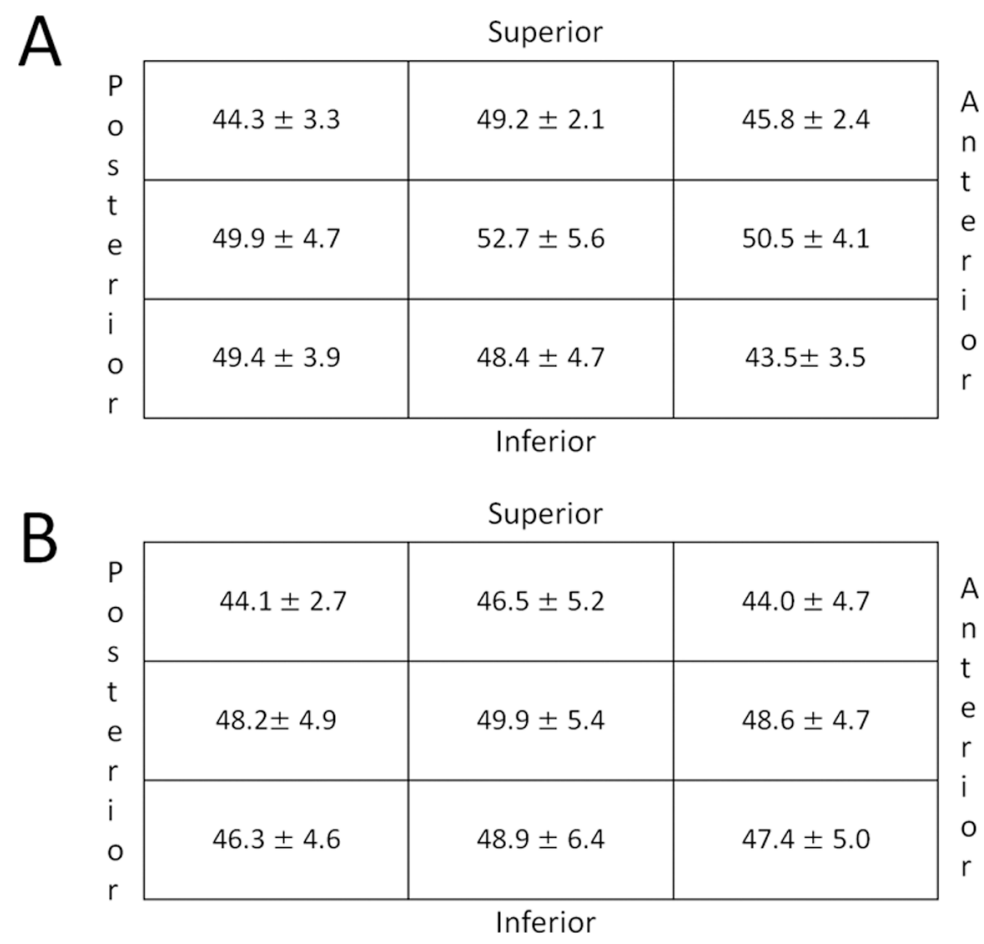

Figure 2.

Average regional T1 $\rho$ values for glenoid cartilage in healthy controls (A) and shoulder dislocation patients (B). Values are mean \pm SD. 
A

\begin{tabular}{|c|c|c|c|}
\hline & \multicolumn{3}{|c|}{ Superior } \\
\hline $\begin{array}{l}P \\
0\end{array}$ & $31.3 \pm 1.0$ & $30.5 \pm 1.3$ & $33.0 \pm 3.7$ \\
\hline e & $38.2 \pm 2.2$ & $40.7 \pm 3.6$ & $40.9 \pm 4.1$ \\
\hline i & $43.8 \pm 2.7$ & $45.8 \pm 3.6$ & $43.2 \pm 4.6$ \\
\hline
\end{tabular}

B

\begin{tabular}{|c|c|c|c|}
\hline & \multicolumn{3}{|c|}{ Superior } \\
\hline $\begin{array}{l}P \\
0\end{array}$ & $35.2 \pm 4.9$ & $33.7 \pm 5.0$ & $35.0 \pm 4.7$ \\
\hline e & $41.5 \pm 3.8$ & $42.5 \pm 2.6$ & $41.8 \pm 3.9$ \\
\hline o & $46.4 \pm 6.2$ & $47.6 \pm 4.3$ & $45.8 \pm 5.1$ \\
\hline
\end{tabular}

Figure 3.

Average regional T1 $\rho$ values for humeral head cartilage in healthy controls (A) and shoulder dislocation patients (B). Values are mean $\pm \mathrm{SD}$. 


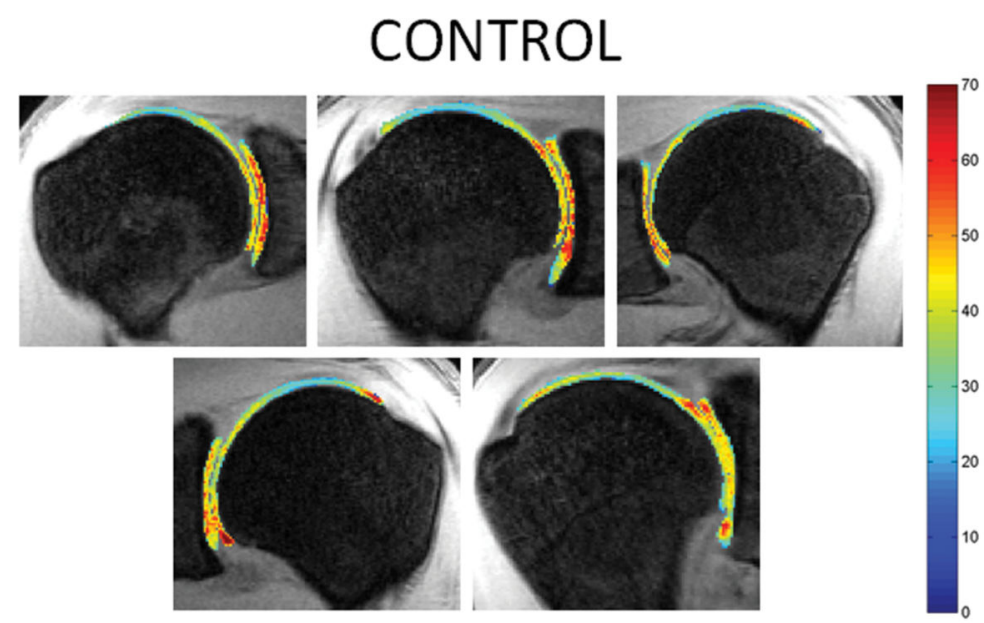

Figure 4.

Representative coronal T1 $\rho$ maps for the five control patients. Higher T1 $\rho$ values in milliseconds are shown in red. 


\section{DISLOCATION}
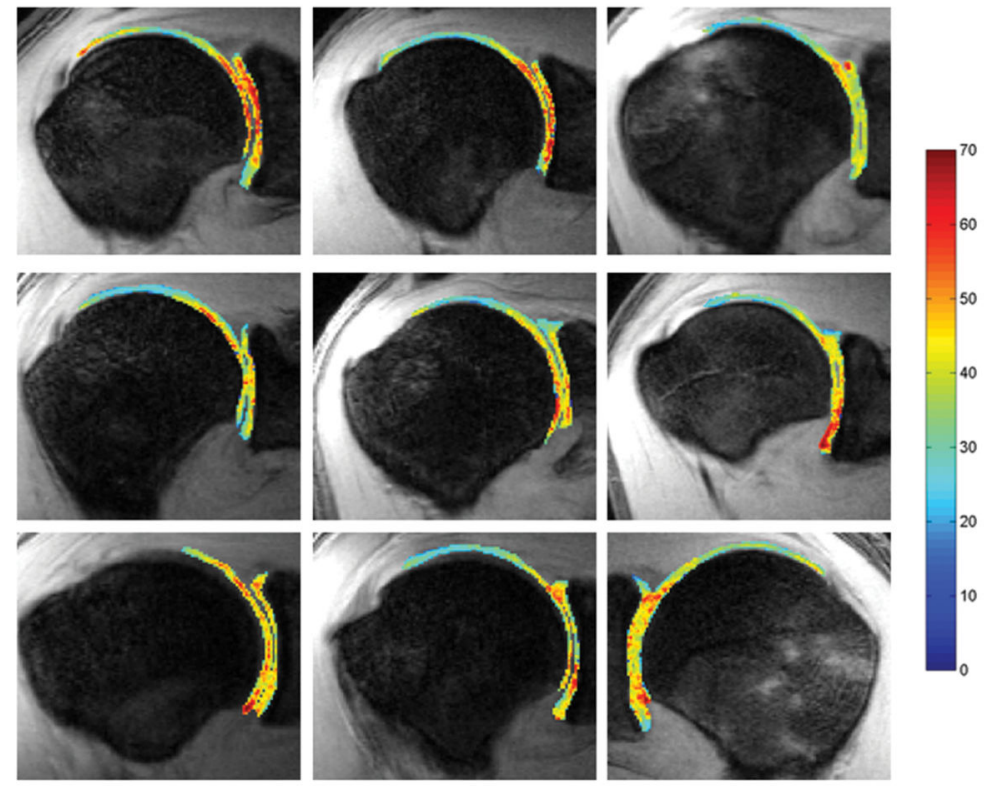

Figure 5.

Representative coronal T1 $\rho$ maps for the nine dislocation patients. Higher T1 $\rho$ values in milliseconds are shown in red. 


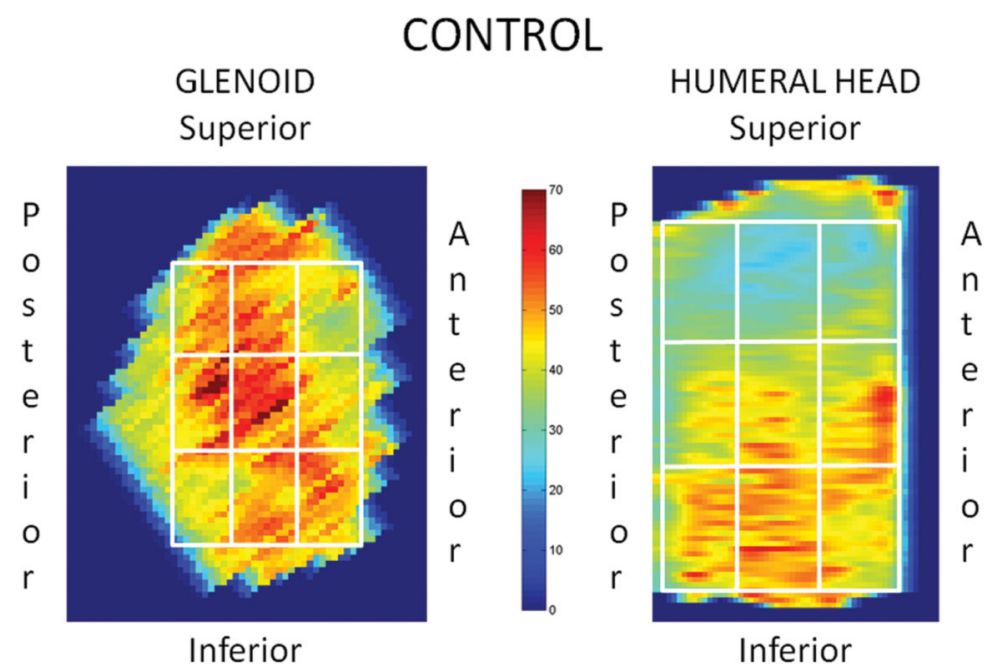

Figure 6.

Sagittal oblique T1 $\rho$ maps with zones overlaid for a healthy control shoulder without prior dislocation. Higher T1 $\rho$ values in milliseconds are shown in red. 


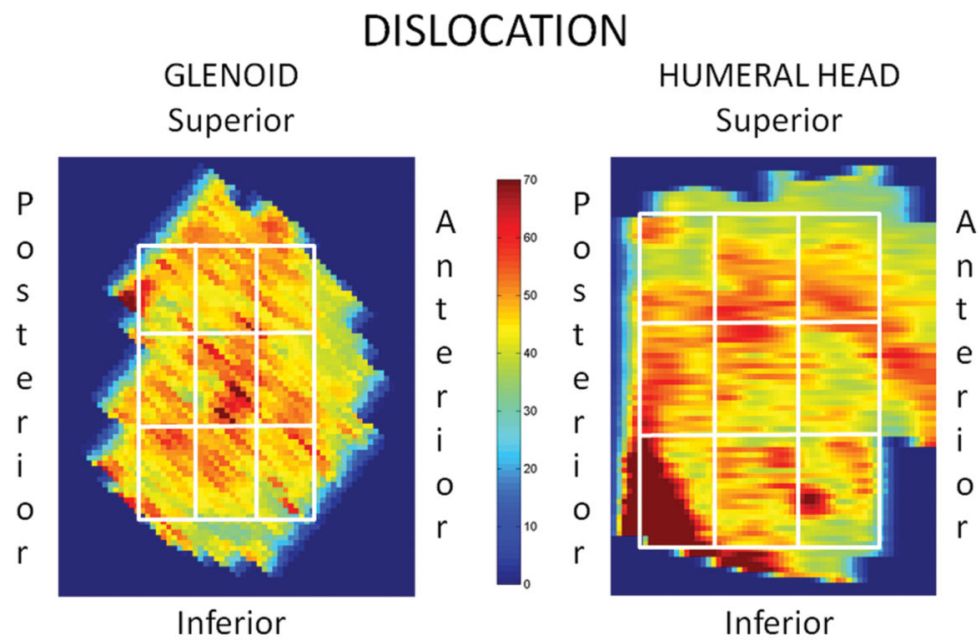

Figure 7.

Sagittal oblique T1 $\rho$ maps with zones overlaid for a dislocation patient showing severe damage to humeral head cartilage. Higher T1 $\rho$ values in milliseconds are shown in red. 